\title{
An adsorption theory of heterogeneous nucleation of water vapour on nanoparticles
}

\author{
A. Laaksonen ${ }^{1,2}$ and J. Malila ${ }^{2}$ \\ ${ }^{1}$ Finnish Meteorological Institute, P.O. Box 503, 00101 Helsinki, Finland \\ ${ }^{2}$ University of Eastern Finland, Department of Applied Physics, P.O. Box 1627, \\ 70211 Kuopio, Finland
}

Correspondence to: A. Laaksonen (ari.laaksonen@fmi.fi)

Received: 17 July 2015 - Published in Atmos. Chem. Phys. Discuss.: 13 August 2015

Revised: 20 November 2015 - Accepted: 25 December 2015 - Published: 15 January 2016

\begin{abstract}
Heterogeneous nucleation of water vapour on insoluble nuclei is a phenomenon that can induce atmospheric water and ice cloud formation. However, modelling of the phenomenon is hampered by the fact that the predictive capability of the classical heterogeneous nucleation theory is rather poor. A reliable theoretical description of the influence of different types of water-insoluble nuclei in triggering the water condensation or ice deposition would help to decrease uncertainty in large-scale model simulations. In this paper we extend a recently formulated adsorption theory of heterogeneous nucleation to be applicable to highly curved surfaces, and test the theory against laboratory data for water vapour nucleation on silica, titanium dioxide and silver oxide nanoparticles. We show that unlike the classical heterogeneous nucleation theory, the new theory is able to quantitatively predict the experimental results.
\end{abstract}

\section{Introduction}

Heterogeneous nucleation of vapours on solid and liquid surfaces is a phenomenon encountered in many natural and industrial systems. For example, cirrus cloud formation by ice deposited from the vapour phase on mineral or other water insoluble aerosols is a climatically important phenomenon initiated by heterogeneous nucleation. Despite decades of research, the classical heterogeneous nucleation theory (CHNT) developed by Fletcher (1958) and its variants that include effects from line tension (Lazaridis, 1993) and transport of adsorbed molecules to the nucleating clusters via surface diffusion (Lee et al., 1998) have been the only available tools for trying to predict the onset of heterogeneous nucleation at given vapour supersaturation and temperature. Unfortunately, the predictions of CHNT fail badly in most cases (Mahata and Alofs, 1975; van der Hage, 1983; Porstendörfer et al., 1985; Chen and Tao, 2000). Molecular level simulations (Zhou et al., 2012; Lupi et al., 2014; Zielke et al., 2015) are becoming a useful tool for understanding the phenomenon; however, they are not a practical alternative when heterogeneous nucleation needs to be predicted within a heavily expensive computing environment such as a global climate model. The purpose of this paper is to show that a recently developed adsorption theory of heterogeneous nucleation (Laaksonen, 2015) modified to account for highly curved substrates is able to quantitatively predict the nucleation of water vapour on different types of nanoparticles.

\section{Theory}

\subsection{The adsorption nucleation model}

The basic assumption behind the new theory is that vapour adsorption on surfaces takes place via formation of molecular clusters around so called active sites. In formulating the theory, we assume that all clusters have similar size, and that the distance between two neighbouring active sites is constant. In reality, the situation is of course very dynamic, with constant evaporation, growth, and coalescence leading to a cluster size distribution. However, in equilibrium, there has to be a well-defined average cluster size that does not evolve with time when temperature and relative humidity are fixed. Likewise, if a snapshot of the situation is taken, there must 
be an average spacing between the clusters that also remains constant.

The clusters are modelled as spherical caps of liquid drops, characterized by a contact angle $\Theta$. As the saturation ratio $S$ of the vapour increases, the droplets grow in equilibrium with the vapour. The equilibrium condition for a droplet is modelled using the Frenkel-Halsey-Hill (FHH) theory of multilayer adsorption (Frenkel, 1946; Halsey, 1948; Hill, 1949), modified by the Kelvin equation that accounts for the influence of the droplet curvature on its vapour pressure. Mathematically, the equilibrium condition is given as

$\ln S=-\frac{A}{\overline{N_{\mathrm{d}}^{B}}}+\frac{2 \gamma v}{k T R}$,

where $A$ and $B$ are FHH-parameters that can be determined by conventional adsorption measurements, $\gamma$ is surface tension, $v$ is volume of the adsorbed molecule (taken to equal liquid-phase molecular volume), $k$ is the Boltzmann constant, $T$ is temperature, and $R$ is radius of the spherical liquid cap, and $N_{\mathrm{d}}$ is the number of monolayers in the water droplet. Note that van der Hage (1983) already presented an equation similar to Eq. (1), but without defining the mathematical form of the adsorption term, while in his subsequent paper (van der Hage, 1984) only a case with a uniform adsorbed layer was considered.

The FHH theory is based on the idea of a "potential field at the surface of a solid into which adsorbate molecules "fall" " (Adamson and Gast, 1997). In the original FHH equation $\ln S=-A N^{-B}$, the term $N^{-B}$ describes how the potential field decays with distance. We therefore define the number of monolayers as a ratio of adsorption layer thickness to monolayer thickness rather than as a ratio of adsorption layer volume to monolayer volume (which is the usual definition). With flat surfaces these two definitions are identical, but that is not the case with curved surfaces. Thus, $N_{\mathrm{d}}=\delta / \delta_{\mathrm{m}}$ with $\delta$ denoting the distance between the substrate and the droplet surface, and the monolayer thickness $\delta_{\mathrm{m}}=v / \sigma$ with $\sigma$ the cross-sectional area of an adsorbed water molecule having a volume $v$.

Because $\delta$ is not constant in the case of a droplet on a substrate, an average over $N_{\mathrm{d}}^{B}$ needs to be taken in Eq. (1), as denoted by the overbar. Previously (Laaksonen, 2015), a relation between $N_{\mathrm{d}}$ and $R$ was given in the case of a flat surface, where the approximation $\overline{N_{\mathrm{d}}^{B}} \approx{\overline{N_{\mathrm{d}}}}^{B}$ was made in order to derive analytical expressions. When the values of adsorption parameters are being determined based on experimental adsorption data (see below), this approximation is in practice necessary because the value of $B$ is initially unknown, and the macroscopic surface coverage $N$ (which is an average over a large number of droplets on the surface) can only be related to $\overline{N_{\mathrm{d}}}$ and not to $\overline{N_{\mathrm{d}}^{B}}$. However, with nucleation calculations the value of $B$ is known, and it is possible to calculate $\overline{N_{\mathrm{d}}^{B}}$ for a single droplet numerically.
With a spherical seed particle, the situation is as shown in Fig. 1. The average over $\delta^{B}$ is given by

$\overline{\delta^{B}}=[1-\cos \Phi]^{-1} \int_{0}^{\Theta} \delta^{B} \sin \alpha \mathrm{d} \alpha$

with

$$
\begin{aligned}
& \delta=-R_{\mathrm{p}}+R \cos \beta \sqrt{(R \cos \beta)^{2}-R^{2}+d^{2}} \\
& d=\sqrt{R_{\mathrm{p}}^{2}+R^{2}-2 R_{\mathrm{p}} R \cos \Theta} \\
& \cos \Phi=\frac{\left(R_{\mathrm{p}}-R \cos \Theta\right)}{d} \\
& \cos \beta=\frac{\left(R_{\mathrm{p}}+\delta-d \cos \alpha\right)}{R} .
\end{aligned}
$$

It is now possible to compute $S$ as a function of $R$ using Eq. (1) with $\overline{N_{\mathrm{d}}^{B}}=\overline{\delta^{B}} / \delta_{\mathrm{m}}^{B}$ evaluated numerically from Eqs. (2)-(6). The curve shows a maximum at the critical supersaturation $S^{*}$ (and critical radius $R^{*}$ ), marking the onset of heterogeneous nucleation. (Alternatively, if one makes the approximation $\overline{N_{\mathrm{d}}^{B}} \approx{\overline{N_{\mathrm{d}}}}^{B}$, the critical supersaturation can be computed from an equation derived in the Appendix. According to our calculations, the resulting error is minor at least for $B$ values up to 3.)

As was pointed out in Laaksonen (2015), nucleation can also take place via coalescence of growing clusters. That is, if the distance between active sites $s$ is sufficiently small, the surface of the nanoparticle may be filled with clusters that coalesce into a uniform liquid film already before they have reached their critical sizes. In practical calculations, we assume that the coalescence transition takes place when the contact area of a cluster with the nanoparticle becomes equal to the square of the average distance between the clusters, i.e. $2 \pi R_{\mathrm{p}}^{2}(1-\cos \Phi)=s^{2}$.

However, the coalescence does not necessarily lead to immediate nucleation. The FHH activation theory (Sorjamaa and Laaksonen, 2007) can be used to calculate the critical supersaturation $S_{\mathrm{U}}^{*}$ of a nanoparticle in the case of zero contact angle, i.e. in a situation where a uniform liquid film is growing on the nanoparticle. In practice, $S_{\mathrm{U}}^{*}$ can be calculated by finding the maximum of $\ln S=-A N^{-B}+(2 \gamma v) /(k T R)$ with $N=\left(R-R_{\mathrm{p}}\right) /\left(2 \delta_{\mathrm{m}}\right)$. Nucleation is immediate if the coalescence takes place above $S_{\mathrm{U}}^{*}$, but otherwise it is delayed until $S_{\mathrm{U}}^{*}$ is reached. Thus, there are three different ways for the nucleation to take place, which we call cluster nucleation (nucleation of single clusters reaching their critical sizes, taking place at $S_{\mathrm{Cl}}^{*}$ ), coalescence nucleation (taking place at $S_{\mathrm{Co}}^{*}$ which is located between $S_{\mathrm{U}}^{*}$ and $S_{\mathrm{Cl}}^{*}$ ), and uniform film nucleation (taking place at $S_{\mathrm{U}}^{*}$ ). In practice, our computer code calculates the equilibrium RH for a spherical cap whose size is increased slightly at each step until a maximum $\mathrm{RH}$ is reached; this point represents cluster nucleation. However, at each step the computer code also checks whether the coalescence transition takes place. If that happens, the code then 


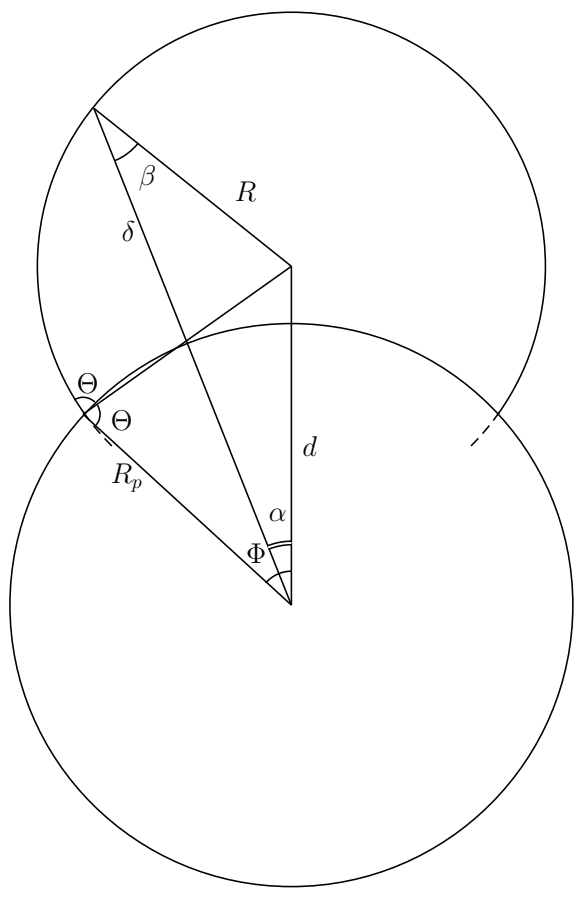

Figure 1. A schematic of a droplet (radius $R$ ) on a nanoparticle (radius $R_{\mathrm{p}}$ ). The contact angle is $\Theta$.

checks whether the RH is above or below the critical supersaturation for uniform film nucleation. If above, we report both the critical supersaturation for the uniform film nucleation and that for the coalescence nucleation. If below, we only report the critical supersaturation for the uniform film nucleation.

\subsection{Determination of the adsorption parameters}

In order to connect the theory to adsorption experiments (from which the adsorption parameter values can be obtained), a relation is needed between the droplet size and the macroscopically observable surface coverage, $N$. The equations below apply for adsorbent surfaces that are sufficiently flat so that their curvature is essentially zero, as is the case with the adsorption experiments considered here (Naono et al., 1994; Every et al, 1961; Kuroda et al., 1997). In general, it is quite rare that adsorption measurements are made with nanoparticles, but in such cases, adsorbent curvature should of course be taken into account. The basic assumption of the theory is that all droplets on the substrate have the same radius, and their average distance is $s$. The macroscopic surface coverage (on a flat substrate) is $N=\delta / \delta_{\mathrm{m}}=V / V_{\mathrm{m}}$, where the $V$ 's denote volumes of the adsorbed layer and a monolayer, respectively, and the adsorbed layer volume $V$ represents a sum of the volumes of all droplets on the surface. Making the approximation $\overline{N_{\mathrm{d}}^{B}} \approx{\overline{N_{\mathrm{d}}}}^{B}$, Laaksonen (2015) translated Eq. (1) into

$$
\begin{gathered}
\ln S=-A\left[\frac{\pi \beta^{2}}{\varepsilon^{2} N}\right]^{\frac{B}{3}}+\frac{2 \gamma}{3 k T}\left[\frac{\pi \varepsilon \beta^{2}}{N}\right]^{\frac{1}{3}}, \quad \Theta \leq 90^{\circ} \\
\ln S=-A\left(\frac{\varepsilon}{b}\right)^{B}\left[\frac{\pi g(\Theta)}{3 s^{2} N}\right]^{\frac{B}{3}}+\frac{2 \gamma v}{3 k T}\left[\frac{\pi g(\Theta)}{3 s^{2} N}\right]^{\frac{1}{3}}, \\
\Theta>90^{\circ} .
\end{gathered}
$$

with $\beta=3 v \sin \Theta / s, \varepsilon=\sigma f(\Theta), g(\Theta)=4-(1+\cos \Theta)^{2}$ $(2-\cos \Theta)$, and $f(\Theta)=(1-\cos \Theta)^{2}(2+\cos \Theta) / \sin ^{2} \Theta$, if $\Theta \leq 90^{\circ} ; f(\Theta)=2-3 \cos \Theta$, if $\Theta>90^{\circ}$.

In order to determine the adsorption parameters from experimental data, we make use of so-called FHH plot, i.e. a diagram of $\ln (-\ln S)$ vs. $\ln N$. When the adsorbent material is sufficiently non-porous, and the contact angle is relatively low, the experimental data can be divided into two approximately linear parts. The lower linear part corresponds to droplet-wise adsorption, and the upper linear part corresponds to adsorption into a uniform liquid film after the surface has been filled by the droplets. As droplets grow in three dimensions and liquid films in one dimension, the slopes of the two linear parts of the data differ by a factor of three. Figure 2 shows an FHH plot for titanium dioxide, and other examples can be seen in Laaksonen (2015). Note that at very low coverages, the data often deviate from the theoretical curves for reasons that may be related, e.g. to the complex microstructures of the adsorbents not captured by the present theory. Furthermore, the transition between the two linear regimes is usually less sharp than predicted by the theory, obviously due to droplet coalescence taking place over some range of relative humidities rather than at one specific value. However, these features do not prevent the determination of the experimental adsorption parameters.

When the contact angle is large enough, the Kelvin effect tends to make the lower part of the FHH plot curved rather than linear. With sufficiently hydrophobic surfaces, the experimental adsorption data may not even reach the uniform film regime as that only occurs very close to $100 \% \mathrm{RH}$ or higher. An example can be seen in Fig. 3 .

In practice, the experimental adsorption parameters are determined as follows. First, it is checked whether a portion of the upper part of the adsorption data in the FHH plot is clearly in the multilayer (or uniform film) regime and aligned linearly. If this is the case, parameters $A$ and $B$ can be determined by fitting the classical FHH equation $\ln S=-A N^{-B}$ to the data. After that, $s$ and $\Theta$ (in case the contact angle has not been measured) can be obtained by fitting Eq. (8) to the rest of the data. However, when the contact angle is large and the adsorption data do not extend to the multilayer regime, all of the parameters (i.e. $A, B$, and $s$, as well as $\Theta$ if it is not known a priori) need to be obtained from a best fit of either Eq. (7) or Eq. (8) to the data set. 


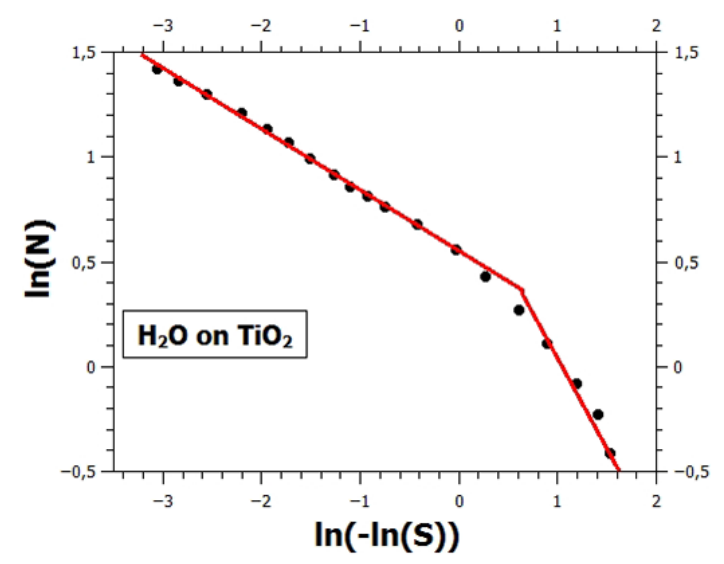

Figure 2. Data of water vapour adsorption on $\mathrm{TiO}_{2}$ (black dots) (Every et al., 1961) and model fit (red line).

\subsection{The classical nucleation theory}

According to the classical heterogeneous nucleation theory (Fletcher, 1958), the free energy of formation of a liquid cluster on a solid surface is given by

$\Delta G=\frac{16 \pi v^{2} \gamma^{3} h(\Theta, x)}{3}$,

where $x=R_{\mathrm{p}} / R$, and the form factor $h(\Theta, x)=[1-$ $\left.\cos ^{3} \Psi+x^{3}\left(2-3 \cos \Phi+\cos ^{3} \Psi\right)+3 x^{2} \cos \Theta(\cos \phi-1)\right]$ with $\cos \Psi=-\left(R-R_{\mathrm{p}} \cos \Theta\right) / d$.

The classical heterogeneous nucleation rate (in units $\mathrm{m}^{-2} \mathrm{~s}^{-1}$ ) is given by

$J=K \exp \left(-\Delta G^{*} / k T\right)$,

where $K$ is a kinetic prefactor that depends rather weakly on temperature and saturation ratio of the nucleating vapour, and the free energy of formation $\Delta G^{*}$ is located by finding a maximum of $\Delta G$ as a function of $R$. The kinetic prefactor can be approximated by a constant of $10^{29}$ (Fletcher, 1958).

Once the heterogeneous nucleation rate is known, the probability of nucleation of a seed particle within time $t$ is $P=1-\exp \left(4 \pi R_{\mathrm{p}}^{2} J t\right)$. We apply the customary definition for nucleation onset condition: it is the saturation ratio at which half of the aerosol population is nucleated, i.e. $P=0.5$. The nucleation time is given by experimental conditions: for $\mathrm{SiO}_{2}$ and $\mathrm{TiO}_{2}$ particles $t=360 \mathrm{~s}$ (Chen et al., 1998), and for $\mathrm{Ag}_{2} \mathrm{O}$ particles $t=5 \mathrm{~ms}$ (Porstendörfer et al., 1985).

\section{Results and discussion}

In order to test the new theory against nucleation experiments, we selected three different data sets with $\mathrm{SiO}_{2}(\mathrm{Chen}$ and Tao, 2000), $\mathrm{TiO}_{2}$ (Chen and Tao, 2000) and $\mathrm{Ag}_{2} \mathrm{O}$ (Porstendörfer et al., 1985) nanoparticles as heterogeneous nuclei for water vapour condensation. Nucleation experiments have
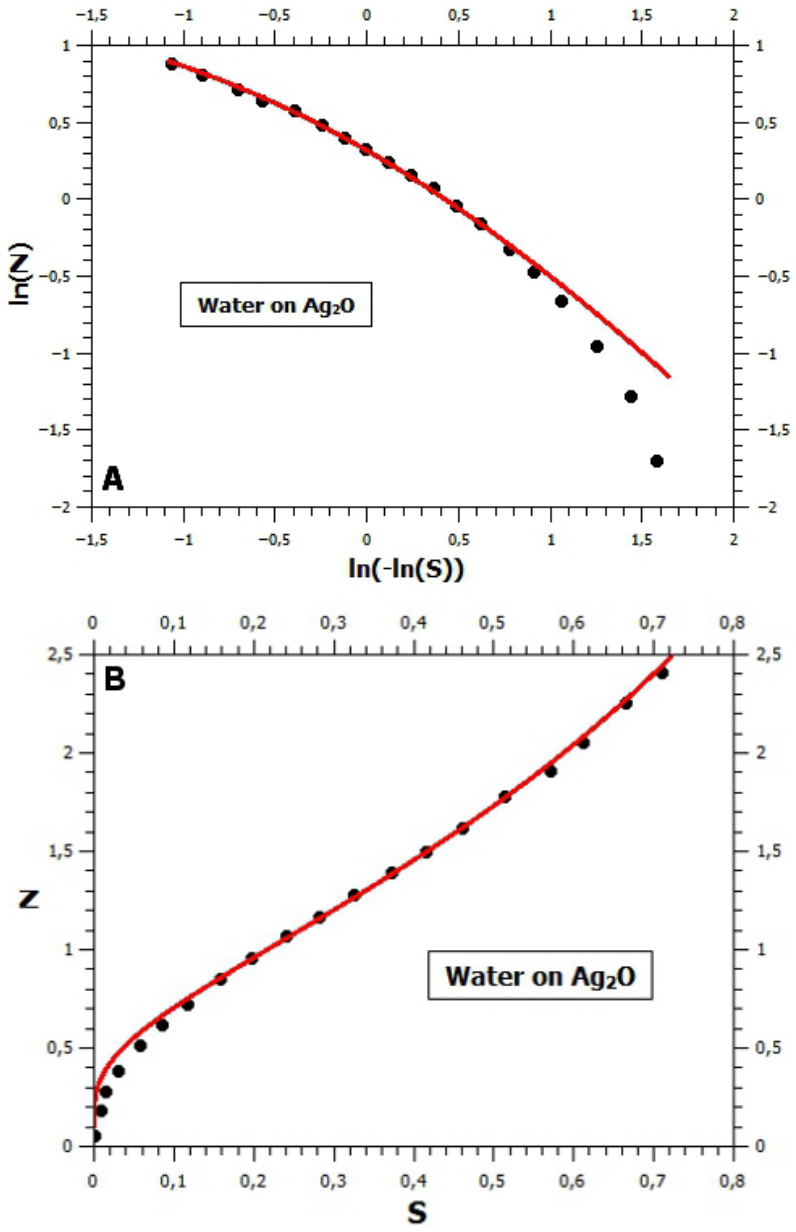

Figure 3. Adsorption of water on silver oxide shown in an FHHplot (a) and on linear scales (b). The data (black dots) have been measured by Kuroda et al. (1997), and the line is calculated using Eq. (8) with the $A, B$, and $s$-parameters optimized using non-linear least squares as described in the text.

been done also with other types of nanoparticles and vapours (e.g. Chen et al., 1998, 1999; Winkler et al., 2008); however, the selected systems were the only ones for which we found both adsorption and contact angle data so that the parameters of the theory could be determined. Note that both Porstendörfer et al. (1985) and Wagner et al. (2003) refer to silver nanoparticles instead of $\mathrm{Ag}_{2} \mathrm{O}$. Nevertheless, the surface of silver nanoparticles can be rapidly oxidized if the carrier gas (compressed air in the experiments of Porstendörfer et al., 1985) contains trace amounts of hydrogen sulfide. As there exists adsorption data for water on silver oxide but none (that we are aware of) for water on silver, we test the theory assuming that the particles' surfaces were oxidized to $\mathrm{Ag}_{2} \mathrm{O}$. Below, we first describe how the adsorption parameter values were obtained for the three systems, and then compare theoretical and experimental onset supersaturations for heterogeneous nucleation. 
Table 1. Experimental parameters used for the three systems. $A, B$, and $s$ were obtained by fitting to the adsorption data obtained from Ref.

\begin{tabular}{lrrrrrrrr}
\hline System & Ref. & $T(\mathrm{~K})$ & $A$ & $B$ & $\Theta\left({ }^{\circ}\right)$ & $s(\mathrm{~nm})$ & $\sigma\left(\AA^{2}\right)^{\mathrm{a}}$ & $\gamma\left(\mathrm{Nm}^{-1}\right)$ \\
\hline $\mathrm{H}_{2} \mathrm{O}-\mathrm{SiO}_{2}$ & $\mathrm{~b}$ & 303 & 1.88 & 1.54 & $20^{\mathrm{c}}$ & 1.61 & 31.6 & 0.071 \\
$\mathrm{H}_{2} \mathrm{O}-\mathrm{TiO}_{2}$ & d & 298 & 6.68 & 3.44 & $16^{\mathrm{c}}$ & 10.5 & 10.3 & 0.072 \\
$\mathrm{H}_{2} \mathrm{O}-\mathrm{Ag}_{2} \mathrm{O}$ & $\mathrm{e}$ & 298 & 14.88 & 2.09 & $90^{\mathrm{f}}$ & 1.09 & 19.0 & 0.072 \\
\hline
\end{tabular}

${ }^{a}$ The cross-sectional areas were obtained from the experimental references; ${ }^{b}$ Naono et al. (1994);

${ }^{\mathrm{c}}$ Chen and Tao (2000); ${ }^{\mathrm{d}}$ Every et al. (1961); ${ }^{\mathrm{e}}$ Kuroda et al. (1997); ${ }^{\mathrm{f}}$ Wagner et al. (2003).

\subsection{Determination of adsorption parameters for $\mathrm{SiO}_{2}$, $\mathrm{TiO}_{2}$, and $\mathrm{Ag}_{2} \mathrm{O}$}

The parameters $A$ and $B$ for water adsorption on $\mathrm{SiO}_{2}$ were obtained from Laaksonen (2015). However, as Chen and Tao (2000) reported the contact angle to be $20^{\circ}$ instead of the $5^{\circ}$ assumed by Laaksonen (2015), the low coverage part of the adsorption data of Naono et al. (1994) needed to be refitted in order to obtain a consistent value for $s$. The resulting parameter values are listed in Table 1.

Figure 2 shows an FHH plot of experimental adsorption data for $\mathrm{TiO}_{2}$ (Every et al., 1961) together with fitted curves. $A$ and $B$ were obtained by fitting the classical FHH equation to the multilayer portion of the data, and $s$ was obtained by fitting Eq. (7) to the sub-monolayer data. The adsorption parameters are given in Table 1.

Wagner et al. (2003) reported the contact angle of water on silver (which we assume to have a surface coating of $\mathrm{Ag}_{2} \mathrm{O}$ ) to be $90^{\circ}$. With such a hydrophobic surface, one would not expect strong multilayer adsorption, and the strong Kelvin effect of the adsorbed droplets should cause the data to fall on a curved line in an FHH plot rather than being linearly aligned (see Laaksonen, 2015). Indeed, as shown in Fig. 3, this is the case with the adsorption data of Kuroda et al. (1997). The adsorption parameters $A$ and $B$ were therefore determined by fitting $S$ from Eq. (7) to data using nonlinear least squares with the Nelder and Mead (1965) method, while parameter $s$ was varied manually to obtain values given in Table 1. (Experimental noise in the data made it impossible to fit all three parameters simultaneously while reproducing the observed isotherm.)

\subsection{Heterogeneous nucleation}

Figure 4 shows experimental (Chen et al., 1998; Chen and Tao, 2000; Chen and Cheng, 2007) and theoretical results of water vapour nucleation on silica nanoparticles. We determined critical supersaturations for both the cluster and uniform film nucleation mechanisms using the new theory. The coalescence of the clusters takes place well below the uniform film nucleation limit (in fact, at subsaturation), and so does not lead to immediate nucleation. Both curves fit the experimental points very well except at the smallest particle sizes. In contrast, the classical theory overestimates the onset supersaturations grossly. Whether the measured size

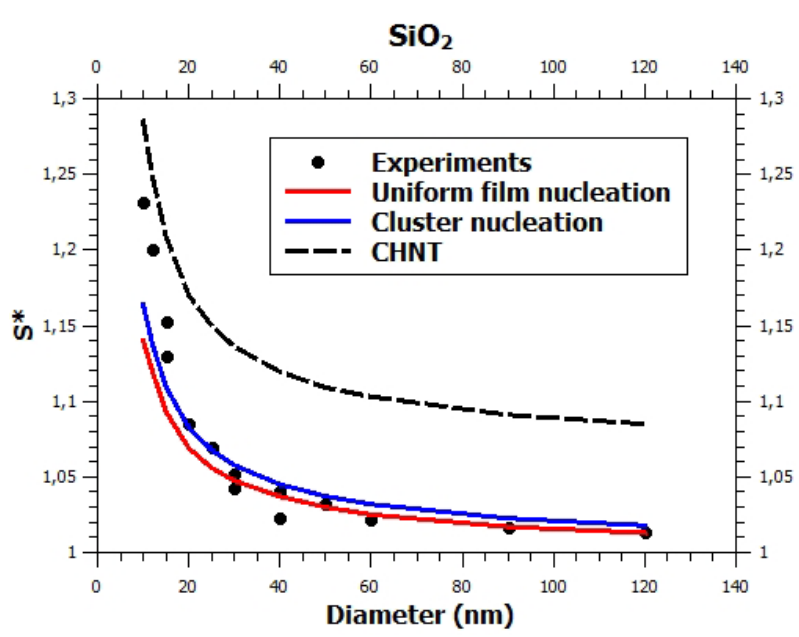

Figure 4. Nucleation of water vapour on silicon dioxide nanoparticles.

dependence at $D_{\mathrm{p}}<20 \mathrm{~nm}$ is real or to some degree an experimental artefact remains somewhat unclear. For example, increasing non-sphericity of the aerosol particles at sizes below $20 \mathrm{~nm}$ would cause the particles to be classified larger than they are in reality, and could thereby create an artificially strong size dependence. The theory of course also assumes spherical particles. Whether the non-sphericity would cause the effective curvature of the particles to increase or to decrease depends on the actual shape; in the former case the theory would underestimate the critical supersaturation, and in the latter case overestimate it.

Figure 5 shows experimental (Chen and Tao, 2000) and theoretical results of water vapour nucleation on titanium dioxide nanoparticles. Again, the cluster coalescence takes place already at subsaturation. Although the new theory predicts somewhat too high onset supersaturations, they are still in much better accord with the experiments than the classical theory. As with silicon dioxide, the difference between the uniform film and cluster nucleation predictions is not very large (note the different $y$ scales in Figs. 4 and 5).

Nucleation of water on silver oxide particles is shown in Fig. 6. In this case, the new theory predicts coalescence nucleation to take place clearly above the uniform film nucleation line. The coalescence nucleation prediction is below the experimental results, however, not much below the un- 


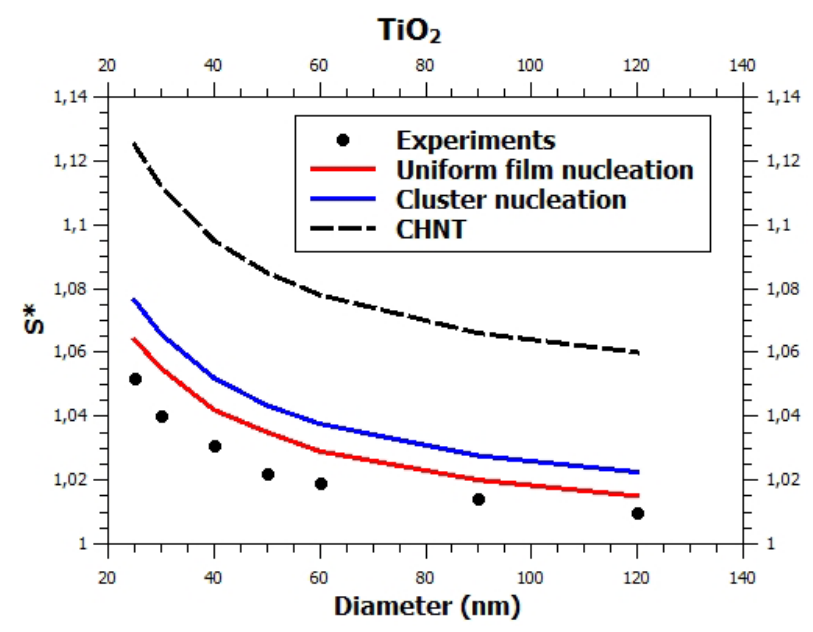

Figure 5. Nucleation of water vapour on titanium dioxide nanoparticles.

certainty limits. Interestingly, the size dependence of the coalescence nucleation is somewhat stronger and better in line with the experiments than that of the cluster nucleation. The prediction of the classical theory is once again well above the experiments, and even though the size dependence of CHNT appears better than that of the new theory (cluster nucleation), it in fact is quite similar which can be seen by lowering the contact angle so that the CHNT curve drops close to the experimental data. This is shown by the dashed line in Fig. 6: using a contact angle of $27^{\circ}$ brings the CHNT curve on top of the coalescence nucleation curve.

Comparison of the experimental and theoretical results in Figs. 4-6 leads to some interesting conclusions. First of all, at relatively low contact angles, the critical supersaturations for cluster nucleation and uniform film nucleation are very close, and therefore, the simpler uniform film nucleation theory (Sorjamaa and Laaksonen, 2007) can quite safely be used e.g. in atmospheric calculations of water nucleation on clay minerals (whose contact angles are just in the same range as those of silicon and titanium dioxides). Furthermore, adsorption data indicate that water forms multilayer films on both of these materials at saturation ratios well below unity, and this fact supports the theoretical result that the cluster coalescence on the nanoparticles occurs at saturation ratios below the critical supersaturation of uniform film nucleation. It is thus very likely that the uniform film nucleation is actually occurring in the cases of $\mathrm{SiO}_{2}$ and $\mathrm{TiO}_{2}$.

In the case of $\operatorname{Ag}_{2} \mathrm{O}$, there is of course considerable uncertainty about the actual contact angle value as we cannot be certain that the surface of the silver used in the contact angle experiment was oxidized. However, the adsorption data (Kuroda et al., 1997) show no indication of a multilayer film formation (which should be observable in the FHH plot of Fig. 3a) as a quite abrupt change of slope, see Laaksonen (2005), although the macroscopic film thickness

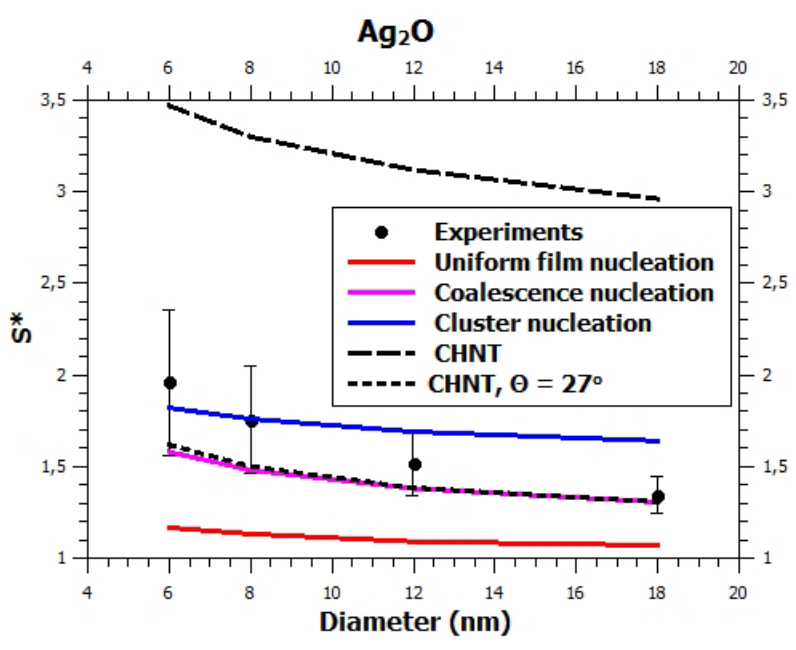

Figure 6. Nucleation of water vapour on silver oxide nanoparticles.

(see Fig. 3b) reaches almost 2.5 monolayers. This is possible only if the adsorbed clusters have quite large contact angles. Moreover, the value of the distance between active sites $s$ determined from the adsorption data will to some extent compensate for the possible error in the contact angle value. For example, if we have assumed a too large $\Theta$, the value of $s$ determined from the adsorption data will be an underestimate compared to reality. These errors will approximately cancel out when either the critical supersaturation of cluster nucleation or that of the coalescence nucleation is calculated. (Note, however, that this holds only for some range of $\Theta$ and $s$, as fitting Eq. (7) (or 8) to the adsorption data becomes impossible if the error in the contact angle is too large, usually a few tens of degrees or more.)

An interesting question relates to the correspondence of the materials used in the adsorption measurements to those applied in the nucleation experiments. The most obvious case in point is the silver nanoparticles, as we cannot really be sure whether their surfaces were oxidized, and to what degree. If they were only partially oxidized (or not at all), then the adsorption parameters we have used are to some degree erroneous, which could explain the discrepancy between the theoretical and experimental results. With titanium dioxide there is some uncertainty as well. The $\mathrm{TiO}_{2}$ nanoparticles used in the nucleation experiments have been analysed with $\mathrm{x}$-ray diffractometry, and found to be anatase-type (Chen et al., 1999). In contrast, the adsorption data taken from Every et al. (1961) represents a "typical curve" of water adsorption on $\mathrm{TiO}_{2}$, and since they used both anatase and rutile types of adsorbent samples, it is somewhat difficult to know how well the adsorption parameters determined based on their data actually correspond to those of pure anatase. Regarding the adsorption properties of silica, it is well known that hightemperature treatment removes hydroxyl groups that provide adsorption sites for water molecules and thus makes silica a less effective adsorbent for water. The silica used in the ad- 
sorption measurements of Naono et al. (1994) was of a grade treated at $800^{\circ} \mathrm{C}$. On the other hand, the silica nanoparticles used in the nucleation experiments were produced by two different techniques: oxidation of $\mathrm{SiCl}_{4}$ at $1000^{\circ} \mathrm{C}$ (Chen et al., 1998), and thermal decomposition of silicon tetraethoxide at $800^{\circ} \mathrm{C}$ (Chen and Tao, 2000; Chen and Cheng, 2007). Apparently, the adsorption parameters determined from the data of Naono et al. (1994) are appropriate for these aerosols.

An obvious application for the new theory is atmospheric ice nucleation via the condensation and deposition mechanisms. It would therefore be interesting to study how well the theory captures the measured temperature dependence of heterogeneous nucleation. The temperature dependence of water vapour nucleating on $40 \mathrm{~nm} \mathrm{TiO}_{2}$ particles was measured by Chen and Tao (2000). They showed the experimental temperature dependence to be stronger than that predicted by the CHNT, and our calculations (not shown) reveal the same to be true with the new theory. However, the temperature dependencies of the adsorption parameters are unknown (they are in principle straightforward to determine, but adsorption measurements at two different temperatures are required), so that nothing conclusive can be said based on our calculations. Regarding silver nanoparticles, Kupc et al. (2013) showed experimentally an anomalous temperature dependence for water vapour nucleation: decreasing critical supersaturation with decreasing temperatures between 278 and $262 \mathrm{~K}$ (usually the temperature dependence of $S^{*}$ is monotonously increasing as $T$ decreases). Very interestingly, Kuroda et al. (1997) have made adsorption measurements as a function of temperature at exactly the same interval, and their data appear to have some unusual features (which the authors attribute to a continuous phase change in the adsorbed layer at around $278 \mathrm{~K}$ ). Unfortunately they made measurements for four constant surface coverages only, which makes it somewhat challenging to tease the actual temperature dependencies of the adsorption parameters out of their data. This exercise will therefore be left to the future.

\section{Conclusions}

A new theory of heterogeneous nucleation on nanoparticles was derived, based on a combination of the FHH adsorption equation and the Kelvin equation. It was assumed that the nucleating clusters can be represented as spherical caps that have a contact angle $\Theta$ with the nanoparticle surface, and reside on active sites located at a distance $s$ apart. It was pointed out that the new theory leads to three different nucleation mechanisms, which were named cluster nucleation, coalescence nucleation, and uniform film nucleation. In the first mechanism, individual clusters grow past their critical sizes, and start collecting vapour by spontaneous condensation. In the second mechanisms, growing clusters fill the nanoparticle surface at a saturation ratio lower than the critical $S$ for cluster nucleation, which again leads to spontaneous growth. In the third mechanism, cluster coalescence also takes place, but at such a low saturation ratio that activation to growth is not possible before the $S$ is increased to the critical supersaturation predicted by the FHH activation theory (Sorjamaa and Laaksonen, 2007) which assumes a zero contact angle.

The new theory was tested for water nucleation on three different nanoparticle types $\left(\mathrm{SiO}_{2}, \mathrm{TiO}_{2}\right.$, and $\mathrm{Ag}_{2} \mathrm{O}$ particles). The FHH adsorption parameters and distances between active sites were determined using adsorption isotherms found in the literature, and the contact angles were obtained from the experimental nucleation papers. With silicon and titanium dioxides, the critical supersaturations calculated with the cluster nucleation and uniform film nucleation mechanisms are very close; nevertheless, it is likely that with these particles nucleation occurs via uniform film nucleation. With $\mathrm{SiO}_{2}$ the theoretical critical supersaturations are in excellent agreement with the experimental results except at the smallest particle sizes $\left(D_{\mathrm{p}}<20 \mathrm{~nm}\right)$. With $\mathrm{TiO}_{2}$ the new theory predicts slightly too high critical supersaturations, possibly influenced by somewhat inaccurate adsorption parameters. Unlike the new theory, CHNT predicts much too high critical supersaturations for both silicon and titanium dioxide.

In the case of silver oxide, there is some uncertainty to the degree of oxidation of the surfaces of both the silver particles used in the nucleation experiments, and of the silver used in the contact angle experiment. Nevertheless, with the assumption that in both cases the surfaces were fully oxidized, the theoretical prediction is in very good agreement with the experiments. Interestingly, the size dependence of the coalescence nucleation is stronger and better in accordance with the experimental observations than that of the cluster nucleation mechanism. As with the two other substances, CHNT overestimates the critical supersaturations of the $\mathrm{Ag}_{2} \mathrm{O}$ nanoparticles quite badly.

In the future, we aim to test the temperature dependence of the new theory, and extend the calculations to atmospherically relevant particle types. 


\section{Appendix A: Derivation of $\bar{\delta}$}

As mentioned in the text, in order to connect the derived theory into experimental adsorption data, the assumption $\overline{N_{\mathrm{d}}} \approx{\overline{N_{\mathrm{d}}}}^{B}=\left(\bar{\delta} / \delta_{\mathrm{m}}\right)^{B}$ is needed. For $\bar{\delta}$ we can derive an analytic expression, which is given here for cases with $\Theta \leq 90^{\circ}$.

To help integration, we switch to a new radial coordinate system with primed variables (complementary angles) depicted in Fig. A1. Applying the cosine law to the triangles, we get two equations, one for $d$ (Eq. 4) and another containing $\delta$,

$$
\begin{aligned}
R^{2}= & d^{2}+\left(R_{\mathrm{p}}+\delta\left(\alpha^{\prime}\right)\right)^{2}-2 d\left(R_{\mathrm{p}}+\delta\left(\alpha^{\prime}\right)\right) \cos \left(\frac{\pi}{2}-\alpha^{\prime}\right) \\
= & R^{2}+2 R_{\mathrm{p}}^{2}-2 R R_{\mathrm{p}} \cos \Theta+2 R_{\mathrm{p}} \delta\left(\alpha^{\prime}\right)+\delta\left(\alpha^{\prime}\right)^{2} \\
& -2 d R_{\mathrm{p}} \sin \alpha^{\prime}-2 d \delta\left(\alpha^{\prime}\right) \sin \alpha^{\prime} .
\end{aligned}
$$

Assuming now that $\Theta \leq \pi / 2$, we get from Eq. (A1) $\Phi^{\prime}=$ $\arcsin \left(R_{\mathrm{p}}-R \cos \Theta\right) / d$, and also

$$
\begin{aligned}
\delta\left(\alpha^{\prime}\right)= & d \sin \alpha^{\prime}-R_{\mathrm{p}}+\left(d^{2} \sin ^{2} \alpha^{\prime}\right. \\
& \left.+2 R R_{\mathrm{p}} \cos \Theta-R_{\mathrm{p}}^{2}\right)^{\frac{1}{2}} .
\end{aligned}
$$

To obtain the proper average, we need to weight $\delta\left(\alpha^{\prime}\right)$ with the surface area of the spherical segment between $\alpha^{\prime}$ and $\alpha^{\prime}+$ $\mathrm{d} \alpha^{\prime}$, i.e. $\mathrm{d} A=\pi R_{\mathrm{p}}^{2} \cos \alpha^{\prime} \mathrm{d} \alpha^{\prime}$ (shaded strip in Fig. A1), and integrate from $\Phi^{\prime}$ to $\pi-\Phi^{\prime}$, or, exploiting the symmetry, from $\Phi^{\prime}$ to $\pi / 2$ :

$$
\begin{aligned}
\bar{\delta}= & \frac{\int_{\Phi^{\prime}}^{\frac{\pi}{2}} \delta\left(\alpha^{\prime}\right) \mathrm{d} A\left(\alpha^{\prime}\right)}{\int_{\Phi^{\prime}}^{\frac{\pi}{2}} \mathrm{~d} A\left(\alpha^{\prime}\right)} \\
= & \frac{1}{\int_{\Phi^{\prime}}^{\frac{\pi}{2}} \cos \alpha^{\prime} \mathrm{d} \alpha^{\prime}} \int_{\Phi^{\prime}}^{\frac{\pi}{2}}\left[d \sin \alpha^{\prime}-R_{\mathrm{p}}+\left(d^{2} \sin ^{2} \alpha^{\prime}\right.\right. \\
& \left.\left.+2 R R_{\mathrm{p}} \cos \Theta-R_{\mathrm{p}}^{2}\right)^{\frac{1}{2}}\right] \cos \alpha^{\prime} \mathrm{d} \alpha^{\prime} \\
= & \frac{d}{2} \frac{\int_{\Phi^{\prime}}^{\frac{\pi}{2}} \sin 2 \alpha^{\prime} \mathrm{d} \alpha^{\prime}}{\int_{\Phi^{\prime}}^{\frac{\pi}{2}} \cos \alpha^{\prime} \mathrm{d} \alpha^{\prime}}-R_{\mathrm{p}} \\
& +\frac{\int_{\Phi^{\prime}}^{\frac{\pi}{2}}\left(d^{2} \sin ^{2} \alpha^{\prime}+2 R R_{\mathrm{p}} \cos \Theta-R_{\mathrm{p}}^{2}\right)^{\frac{1}{2}} \cos \alpha^{\prime} \mathrm{d} \alpha^{\prime}}{\int_{\Phi^{\prime}}^{\frac{\pi}{2}} \cos \alpha^{\prime} \mathrm{d} \alpha^{\prime}} .
\end{aligned}
$$

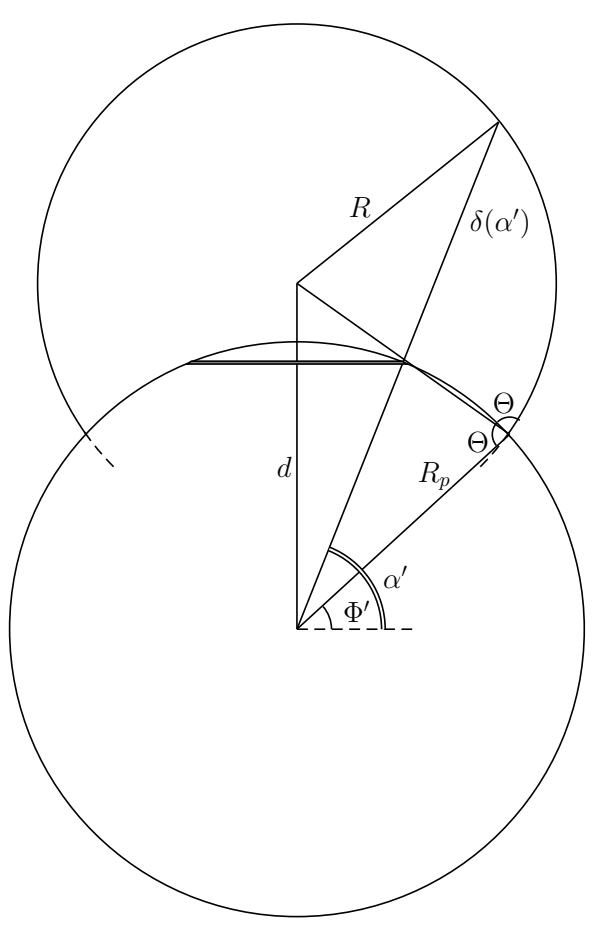

Figure A1. Geometry of the problem revisited.

The first two terms are trivial, and the nominator of the third one can be integrated in parts, resulting in

$$
\begin{aligned}
\bar{\delta}= & \frac{d}{2}\left(1+\sin \Phi^{\prime}\right)-R_{\mathrm{p}} \\
& +\frac{R-\left(d^{2} \sin ^{2} \Phi^{\prime}+2 R R_{\mathrm{p}} \cos \Theta-R_{\mathrm{p}}^{2}\right)^{\frac{1}{2}}}{2\left(1-\sin \Phi^{\prime}\right)} \\
& +\frac{2 R R_{\mathrm{p}} \cos \Theta-R_{\mathrm{p}}^{2}}{2 d\left(1-\sin \Phi^{\prime}\right)} \\
& \times \ln \frac{R+d}{\left(d^{2} \sin ^{2} \Phi^{\prime}+2 R R_{\mathrm{p}} \cos \Theta-R_{\mathrm{p}}^{2}\right)^{\frac{1}{2}}+d \sin \Phi^{\prime}} .
\end{aligned}
$$

Substituting $\Phi^{\prime}=\arcsin \left(R_{\mathrm{p}}-R \cos \Theta\right) / d$ back to Eq. (A4) we get after some algebra

$$
\begin{aligned}
\bar{\delta}= & \left(R^{2} \sin ^{2} \Theta+R_{\mathrm{p}}^{2}\left\{2-\ln \left[(R+d) / R_{\mathrm{p}}\right]\right\}\right. \\
& \left.+d\left[R(1-\cos \Theta)-2 R_{\mathrm{p}}\right]\right)[2(d+R \cos \Theta \\
& \left.\left.-R_{\mathrm{p}}\right)\right]^{-1} .
\end{aligned}
$$


Acknowledgements. This work was supported by the Academy of Finland Centre of Excellence programme (grant no. 272 041).

Edited by: H. Su

\section{References}

Adamson, A. W., and Gast, A. P.: Physical Chemistry of Surfaces, Wiley, New York, USA, p. 625, 1997.

Chen, C.-C. and Cheng, H.-C.: Effects of charge and size on condensation of supersaturated water vapor on nanoparticles of $\mathrm{SiO}_{2}$, J. Chem. Phys., 126, 034701, doi:10.1063/1.2424707, 2007.

Chen, C.-C. and Tao, C.-J.: Condensation of supersaturated water vapor on submicrometer particles of $\mathrm{SiO}_{2}$ and $\mathrm{TiO}_{2}$, J. Chem. Phys., 112, 9967-9977, doi:10.1063/1.481633, 2000.

Chen, C.-C., Guo, M.-S., Tsai, Y.-J., and Huang, C.-C.: Heterogeneous nucleation of water vapor on submicrometer particles of $\mathrm{SiC}, \mathrm{SiO}_{2}$, and naphthalene, J. Colloid Interf. Sci., 198, 354-367, doi:10.1006/jcis.1997.5298, 1998.

Chen, C.-C., Huang, C.-C., and Tao, C.-J.: Heterogeneous nucleation of $n$-butanol vapor on submicrometer particles of $\mathrm{SiO}_{2}$ and $\mathrm{TiO}_{2}$, J. Colloid Interf. Sci., 211, 193-203, doi:10.1006/jcis.1998.6007, 1999.

Every, R. L., Wade, W. H., and Hackerman, N.: Free energy of adsorption, II. The influence of substrate structure in the systems $\mathrm{Al}_{2} \mathrm{O}_{3}$ and $\mathrm{TiO}_{2}$ with $n$-hexane, $\mathrm{CH}_{3} \mathrm{OH}$ and $\mathrm{H}_{2} \mathrm{O}$, J. Phys. Chem., 65, 937-941, doi:10.1021/j100824a011, 1961.

Fletcher, N. H.: Size effect in heterogeneous nucleation, J. Chem. Phys., 29, 572-576, doi:10.1063/1.1744540, 1958.

Frenkel, J.: Kinetic Theory of Liquids, Oxford University Press, London, UK, 332-339, 1946.

Halsey, G.: Physical adsorption on non-uniform surfaces, J. Chem. Phys., 16, 931-945, doi:10.1063/1.1746689, 1948.

Hill, T. L.: Physical adsorption and the free volume model for liquids, J. Chem. Phys., 17, 590, doi:10.1063/1.1747341, 1949.

Kupc, A., Winkler, P. M., Vrtala, A., and Wagner, P.: Unusual temperature dependence of heterogeneous nucleation of water vapor on $\mathrm{Ag}$ particles, Aerosol Sci. Tech., 47, i-iv, doi:10.1080/02786826.2013.810330, 2013.

Kuroda, Y., Watanabe, T., Yoshikawa, Y., Kumashiro, R., Hamano, H., and Nagao, M.: Specific feature of dielectric behavior of water adsorbed on $\mathrm{Ag}_{2} \mathrm{O}$ surface, Langmuir, 13, 38233826, doi:10.1021/1a951505j, 1997.

Laaksonen, A.: A unifying model for adsorption and nucleation of vapors on solid surfaces, J. Phys. Chem. A, 119, 3736-3745, doi:10.1021/acs.jpca.5b00325, 2015.

Lazaridis, M.: The effects of surface diffusion and line tension on the mechanism of heterogeneous nucleation, J. Colloid Interf. Sci., 155, 386-391, doi:10.1006/jcis.1993.1051, 1993.
Lee, Y.-L., Chou, W.-S., and Chen, L.-H.: The adsorption and nucleation of water vapor on an insoluble spherical solid particle, Surf. Sci., 414, 363-373, doi:10.1016/S0039-6028(98)00441-5, 1998.

Lupi, L., Hudait, A., and Molinero, V.: Heterogeneous nucleation of ice on carbon surfaces, J. Am. Chem. Soc., 136, 3156-3164, doi:10.1021/ja411507a, 2014.

Mahata, P. C. and Alofs, D. J.: Insoluble condensation nuclei: the effect of contact angle, surface roughness and adsorption, J. Atmos. Sci., 32, 116-122, doi:10.1175/15200469(1975)032<0116:ICNTEO>2.0.CO;2, 1975.

Naono, H., Hakuman, M., and Nakai, K.: Determination of pore size distribution of mesoporous and macroporous silicas by means of benzene-desorption isotherms, J. Colloid Interf. Sci., 165, 532535, doi:10.1006/jcis.1994.1260, 1994.

Nelder, J. A. and Mead, R.: A simplex method for function minimization, Comput. J., 7, 308-313, doi:10.1093/comjnl/7.4.308, 1965.

Porstendörfer, J., Scheibel, H. G., Pohl, F. G., Preining, O., Reischl, G., and Wagner, P. E.: Heterogeneous nucleation of water vapor on monodispersed $\mathrm{Ag}$ and $\mathrm{NaCl}$ particles with diameters between 6 and $18 \mathrm{~nm}$, Aerosol Sci. Tech., 4, 65-79, doi:10.1080/02786828508959039, 1985.

Sorjamaa, R. and Laaksonen, A.: The effect of $\mathrm{H}_{2} \mathrm{O}$ adsorption on cloud drop activation of insoluble particles: a theoretical framework, Atmos. Chem. Phys., 7, 6175-6180, doi:10.5194/acp-76175-2007, 2007.

van der Hage, J. H. C.: Condensation on insoluble particles, J. Colloid Interf. Sci., 91, 384-390, doi:10.1016/0021-9797(83)90351X, 1983.

van der Hage, J. H. C.: Drop formation on insoluble particles, J. Colloid Interf. Sci., 101, 10-18, doi:10.1016/0021-9797(84)90003$1,1984$.

Wagner, P. E., Kaller, D., Vrtala, A., Lauri, A., Kulmala, M. and Laaksonen, A.: Nucleation probability in binary heterogeneous nucleation of water- $n$-propanol vapor mixtures on insoluble and soluble nanoparticles, Phys. Rev. E, 67, 021605, doi:10.1103/PhysRevE.67.021605, 2003.

Winkler, P., Steiner, G., Vrtala, A., Vehkamäki, H., Noppel, M., Lehtinen, K. E. J., Reischl, G. P., Wagner, P. E., and Kulmala, M.: Heterogeneous nucleation experiments bridging the scale from molecular ion clusters to nanoparticles, Science, 319, 13741377, doi:10.1126/science.1149034, 2008.

Zhou, D., Mi, J., and Zhong, C.: Three-dimensional density functional study of heterogeneous nucleation of droplets on solid surfaces, J. Phys. Chem. B, 116, 14100-14106, doi:10.1021/jp307820w, 2012.

Zielke, S. A., Bertram, A. K., and Patey, G. N.: A molecular mechanism of ice nucleation on model AgI surfaces, J. Phys. Chem. B, 119, 9049-9055, doi:10.1021/jp508601s, 2015. 